\title{
MicroRNA-142-3p inhibits trophoblast cell migration and invasion by disrupting the TGF-ß31/Smad3 signaling pathway
}

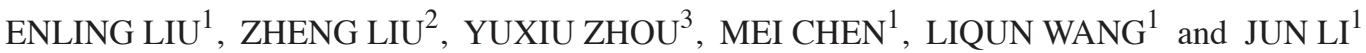 \\ ${ }^{1}$ Department of Obstetrics and Gynecology, Tangshan Worker Hospital, Hebei Medical University, Tangshan, \\ Hebei 063000; ${ }^{2}$ Department of Rheumatology and Immunology, Tianjin General Hospital, Tianjin Medical University, \\ Tianjin 300052; ${ }^{3}$ Department of Rheumatology and Immunology, Tangshan Worker Hospital, \\ Hebei Medical University, Tangshan, Hebei 063000, P.R. China
}

Received September 9, 2017; Accepted July 2, 2018

DOI: $10.3892 / \mathrm{mmr} .2019 .9997$

\begin{abstract}
Insufficient invasion of trophoblasts is known to be associated with preeclampsia (PE) development. Recently, microRNAs (miRNAs) have been reported to serve important roles in the pathogenesis of PE. However, little is known regarding the regulation of trophoblastic invasion by miRNAs. The aim of the present study was to explore the role of miRNAs in trophoblastic invasion and the underlying molecular mechanism. Using a miRNA microarray, miRNAs putatively involved in the pathophysiology of PE were examined between normal and preeclamptic placentas. Validation analysis of miR-142-3p level in placenta specimens was performed using reverse transcription-quantitative polymerase chain reaction (RT-qPCR). Then, the regulation of miR-142-3p on trophoblast cells migration and invasion was evaluated using wound healing and transwell migration assays. Furthermore, the target gene of miR-142-3p and the downstream signaling pathway were also investigated. Microarray analysis and RT-qPCR revealed that miR-142-3p was significantly upregulated in placenta specimens from patients with PE. Its overexpression inhibited trophoblast cell invasion and migration, whereas its knockdown enhanced trophoblast cell invasion and migration. In addition, overexpression of miR-142-3p inhibited the mRNA expression and the activities of matrix metalloproteinase-2 (MMP2) and MMP9, which are closely associated with cell invasion and migration, while inhibition of miR-142-3p had the opposite result. Subsequent analyses demonstrated that transforming growth factor- $\beta 1$ (TGF- $\beta 1$ ) was a direct and functional target of miR-142-3p. Notably, the knockdown of TGF- $\beta 1$ effectively reversed the enhancement of miR-142-3p inhibitor on trophoblast cell invasion and migration. Finally, the present study
\end{abstract}

Correspondence to: Professor Enling Liu, Department of Obstetrics and Gynecology, Tangshan Worker Hospital, Hebei Medical University, 27 Wenhua Road, Tangshan, Hebei 063000, P.R. China

E-mail:2271854732@qq.com

Key words: microRNA-142-3p, pre-eclampsia, trophoblast invasion, HTR8/SVneo cells, transforming growth factor- $\beta 1 / \mathrm{smad} 3$ confirmed that miR-142-3p inhibitor enhanced cell invasion and migration by reactivating the TGF- $\beta 1 / \mathrm{Smad} 3$ signaling pathway. Taken together, the results of the present study suggest that miR-142-3p may serve an important role in human placental development by suppressing trophoblast cell invasion and migration through disruption of the TGF- $\beta 1 / \mathrm{smad} 3$ signaling pathway, suggesting that knockdown of miR-142-3p may provide a novel therapy for PE.

\section{Introduction}

Preeclampsia (PE) is a pregnancy-specific syndrome characterized by the new onset of hypertension and proteinuria after 20 weeks of gestation. PE affects 3-5\% pregnant women and results in high maternal and neonatal morbidity and mortality worldwide $(1,2)$. Despite major efforts have been made in past decades, the available therapeutic options to retard disease progression remain limited. Thus, it is an urgent need to search for a principal therapeutic target for PE.

Normal placentation is believed to play an important role during pregnancy. The process of placentation involves cells derived from the placenta, fetal extravillous trophoblasts (EVTs), invading into the endometrium and one third of the myometrium, as well as the associated spiral arteries under strict temporal and spatial controls (3-5). Subsequently, the spiral arteries were replaced and converted into highly dilated vessels that are able to provide adequate placental perfusion to maintain the growth of fetus (6). There is increasing evidence to support that insufficient trophoblastic invasion is central to the pathogenesis of PE $(7,8)$. However, the molecular mechanism for the regulation of trophoblast invasion remains largely elusive.

MicroRNAs (miRNAs) are a class of small non-coding RNAs which negatively regulate gene expression by binding to the 3'-untranslated regions (3'-UTR) of their target mRNAs for transcript degradation or translational repression (9). miRNAs were recently reported to play functional roles in various cellular processes in almost all diseases, such as proliferation, apoptosis, invasion, and differentiation $(10,11)$. It has been found that the expression profiles of miRNAs were significantly altered in placenta tissues and maternal serum from PE pregnancies $(12,13)$. For example, by screening the expression 
profile of miRNAs in the placentas of PE patients and healthy subjects, Enquobahrie et al (14), identified that eight miRNAs were differentially expressed in the PE placentas, suggesting that aberrant expression of miRNAs may contribute to the pathogenesis of PE.

In the present study, we focused the expression level of miR-142-3p in the placentas of PE patients and studied its role in the invasion and migration of trophoblast cells. Moreover, we also studied the association between miR-142-3p and TGF- $\beta 1$ in the invasion ability of trophoblast cells, as well as the downstream signaling pathway.

\section{Materials and methods}

Tissue samples. The placental tissues used in this study were obtained from women with normal pregnancies $(n=20)$ and patients with PE $(n=20)$ at the Department of Obstetrics and Gynecology, Tangshan Worker Hospital. All experimental protocols were approved by the Ethics Committee of the Tangshan Worker Hospital, He Bei Medical University. All studies were performed in accordance with the ethical guidelines of the Tangshan Worker Hospital, He Bei Medical University. Written informed consent was obtained from all patients.

miRNA expression profiling. Total RNA was isolated from frozen placentas tissue by the miRNeasy mini kit (Qiagen, Ltd., West Sussex, UK) following the manufacturer's protocol. After passing the RNA purity and quantity measurement using the NanoDrop ND-1000 Spectrophotometry (Thermo Fisher Scientific, Inc., Waltham, MA, USA) and Agilent's 2100 Bioanalyzer, samples were labeled with miRCURY ${ }^{\mathrm{TM}} \mathrm{Hy} 3^{\mathrm{TM}} /$ $\mathrm{Hy}^{\mathrm{TM}}$ Power labeling kit and hybridized to the miRCURY LNA $^{\text {TM }}$ Array (v.18.0; Agilent Technologies, Inc., Santa Clara, CA, USA), incubated, washed and scanned in Agilent high resolution microarray scanner. Data was analyzed using Genespring software (Agilent Technologies, Inc.). The heat map of the 54 miRNAs most obvious differences was created using a method of hierarchical clustering by GeneSpring GX, v.7.3 (Agilent Technologies, Inc.).

Reverse transcription-quantitative PCR (RT-qPCR). Total RNA and miRNA were isolated from placentas specimens using the miRNeasy Mini kit (Qiagen GmbH, Hilden, Germany) following the manufacturer's instructions. For the detection of miR-142-3p, miR-142-3p was reverse transcribed using the PrimeScript RT reagent kit (Takara Biotechnology Co., Ltd., Dalian, China) and quantified by RT-qPCR with the TaqMan MicroRNA assay kit (Applied Biosystems; Thermo Fisher Scientific, Inc.). For detection of the TGF- $\beta 1$ mRNA levels, $1 \mu \mathrm{g}$ of total RNA was reverse transcribed using Script ${ }^{\mathrm{TM}}$ cDNA Synthesis kit (Bio-Rad Laboratories, Inc., Hercules, CA, USA), RT-qPCR were performed using SYBR Premix Ex Taq (Takara Biotechnology Co., Ltd., Dalian, China) on an ABI PRISM 7500 Sequence Detection System (Applied Biosystems; Thermo Fisher Scientific, Inc.). U6 and glyceraldehyde 3-phosphate dehydrogenase (GAPDH) were used as normalization control in the expression analysis of miR-142-3p and TGF- $\beta 1$, respectively. The sequences of the primers were as follows: MMP-2 sense 5'-GGCCTCGTATACCGCATCAAT C-3', anti-sense 5'-GGCCTCTCCTGACATTGACCTT-3';
MMP-9 sense 5'-CCCGGACCAAGGATACAG-3', anti-sense 5'-GGCTTTCTCTCGGTACTG-3'; GAPDH sense 5'-ATT GTTGCCATCAATGACCC-3', anti-sense 5'-AGTAGAGGC AGGGATGATGT-3'. Primers for miR-142-3p and U6 snRNA were obtained from GeneCopoeia. The relative expression of RNAs was calculated using the $2^{-\Delta \Delta C q}$ method (15). Each reaction was conducted in triplicate.

Cell culture. The extravillous trophoblast cell line HTR-8/ SVneo was obtained from ATCC and maintained in RPMI 1640 medium (Thermo Fisher Scientific, Inc.) supplemented with $10 \%$ fetal bovine serum (Thermo Fisher Scientific, Inc.). Human 293T cells were purchased from the American Type Culture Collection (Manassas, VA, USA) and maintained in Dulbecco's modified Eagle's medium (Invitrogen; Thermo Fisher Scientific, Inc.) containing 10\% fetal bovine serum (Invitrogen; Thermo Fisher Scientific, Inc.) and 1/100 streptomycin-penicillin mix (Sigma-Aldrich; Merck KGaA, Darmstadt, Germany). The cells were incubated at $37^{\circ} \mathrm{C}$ with $5 \% \mathrm{CO}_{2}$.

Transfection. The miR-142-3p mimics, miR-142-3p inhibitor and negative control (6) were synthesized by GenePharma Biological Technology (Shanghai, China). The TGF- $\beta 1$ siRNA and NC siRNA were purchased from GenePharma Biological Technology. Cell transfection was performed using Lipofectamine 2000 (Invitrogen; Thermo Fisher Scientific, Inc.) according to the manufacturer's instructions. The cells were treated for further experiments $48 \mathrm{~h}$ after transfection.

In vitro invasion and migration assay. For wound healing assay, HTR-8/SVneo cells were transfected with miR-142-3p mimics, miR-142-3p inhibitor and negative control (6) or si-TGF- $\beta 1$ and seeded into 6 -well plates at a density of 1,000 cells/well. After $48 \mathrm{~h}$, when the cells reached $80 \%$ confluence, scratch wounds were wounded with a $10 \mu$ l pipette tip; cells were washed three times with PBS to clear cell debris; and wound gaps were imaged and calculated by Image J software (NIH, Bethesda, MD, USA) at 24 and 48 h. For invasion assay, the invasive ability of trophoblasts was evaluated using a polycarbonate membrane cell culture insert (Costar; Corning Incorporated, Corning, NY, USA) coated with growth factor reduced Matrigel (BD Biosciences, Franklin Lakes, NJ, USA) as previously described (16). The number of invaded cells was calculated by counting five random views under the microscope at 24 and $48 \mathrm{~h}$. The experiment was performed in triplicate and repeated for three times.

Gelatin zymography. Gelatin zymography is an extremely sensitive and useful technique for measuring the relative amounts of active and inactive gelatinase (MMP-2 or MMP-9) in samples. Briefly, samples $(10 \mu \mathrm{l})$ were resuspended in $5 \mathrm{x}$ non-reducing sample buffer (4\% SDS, $20 \%$ glycerol, $0.01 \%$ bromophenol blue and $125 \mathrm{mM}$ Tris- $\mathrm{HCl}$ ), and run on a 10\% SDS-PAGE gel containing $0.5 \mathrm{mg} / \mathrm{ml}$ gelatin without prior denaturation. After electrophoresis, the gels were washed to remove SDS and incubated for 5-10 min at room temperature (RT) in an incubation buffer $\left(50 \mathrm{mM}\right.$ Tris, $5 \mathrm{mM} \mathrm{CaCl}, 1 \mu \mathrm{M} \mathrm{ZnCl}_{2}$, and $1 \%$ Triton $\mathrm{X}-100)$. Next, replace with fresh incubation buffer and incubate for $24 \mathrm{~h}$ at $37^{\circ} \mathrm{C}$. The gels were subsequently stained with 
Coomassie brilliant blue G-250, destained in 30\% methanol, and flooded with $10 \%$ acetic acid to detect gelatinase secretion.

Western blot analysis. Total cell protein was abstracted from cells after transfection using radioimmunoprecipitation (RIPA) lysis buffer (Sigma-Aldrich; Merck KGaA) and the quantity of the protein was determined using a Bicinchoninic Acid (BCA) protein assay kit (Pierce; Thermo Fisher Scientific, Inc.). $40 \mu \mathrm{g}$ of protein samples were subjected to SDS-polyacrylamide gel electrophoresis and transferred onto a PVDF membrane. The membrane was blocked with $5 \%$ nonfat milk. The membrane was then incubated with primary antibodies: Anti-TGF- $\beta 1$ antibody (Rabbit polyclonal, 1:1,000) and anti- $\beta$-actin antibody (Rabbit polyclonal, 1:2,000) (all antibodies were purchased from Abcam, Cambridge, MA, USA), followed by incubation with HRP-anti-rabbit secondary antibody at room temperature for $1 \mathrm{~h}$. Signals were detected using an ECL kit (GE Healthcare, Chicago, IL, USA) according to the manufacturer's protocol. The intensity of protein fragments was quantified with the Quantity One software (v.4.5.0 basic, Bio-Rad Laboratories, Inc.).

Luciferase assays. A cDNA fragment of the TGF- $\beta 1$ 3'-UTR mRNAcontaining the seed sequence of the miR-142-3p-binding site or a mutated binding site was cloned into the pmirGLO dual-luciferase vector (Promega Corporation, Madison, WI, USA). The constructed dual-luciferase vector was co-transfected with 10 pmol of miR-142-3p mimics, miR-142-3p inhibitor or NC into $293 \mathrm{~T}$ cells. The cells were harvested and lysed $24 \mathrm{~h}$ later, and the luciferase activity was measured by the Dual-Luciferase Assay System (Promega Corporation) in accordance to the manufacturer's instructions.

Statistical analysis. Data are presented as the mean \pm standard deviation of three independent experiments. Statistical analysis was performed using GraphPad Prism v.5.0 (GraphPad Software, Inc., San Diego, CA, USA). Differences were analyzed with the Student's t-test between two groups or with one-way analysis of variance followed by Tukey's multiple comparison tests between multiple groups. $\mathrm{P}<0.05$ was considered to indicate a statistically significant difference.

\section{Results}

miR-142-3p was upregulated in placentas from patients with $P E$. In order to identify the changes in miRNA expression involved in the pathogenesis of PE, a miRNA microarray with a pool of miRNAs of frozen placentas from 3 patients with PE and 3 normal pregnancies was performed. Our data revealed that 29 miRNAs were upregulated and 25 miRNAs were downregulated in the PE group (Fig. 1A). Among the aberrantly expressed miRNAs, miR-142-3p was one of the miRNAs being most significantly upregulated. In previous studies, miR-142-3p has been also found to be upregulated in the placenta tissues from PE patients (17-19). Moreover, miR-142-3p expression correlated with the invasiveness of different types of human cancer cells (20-22). Therefore, we chose miR-142-3p for further research. To validate the results of miRNA microarray, the miR-142-3p expression in 20 pairs of placentas from patients with PE and normal pregnancies was determined by RT-qPCR. The results showed that miR-142-3p expression was significantly upregulated in placentas compared with normal placentas tissues (Fig. 1B). These results suggested that miR-142-3p may be involved in the pathogenesis of PE.

Knockdown of miR-142-3p promotes invasiveness of trophoblast cells. To investigate the role of miR-142-3p on migration and invasion of trophoblast cells, HTR-8/SVneo cells derived from human extravillous trophoblast, which is widely used as in vitro models for studies of trophoblast invasion (23), were transiently transfected with miR-142-3p mimics, miR-142-3p inhibitor or negative control (6), then the invasion and migration were measured by wound healing assay and transwell invasion assay at 24 and $48 \mathrm{~h}$. As shown in Fig. 2A and B, miR-142-3p overexpression significantly suppressed the migration and invasion of HTR-8/SVneo cells. In contrast, knockdown of miR-142-3p significantly increased the migration and invasion of HTR-8/SVneo cells (Fig. 2C and D). It is well known that the MMPs activity plays an important role in trophoblast invasion $(24,25)$. For this reason, we measured the effect of miR-142-3p on the mRNA levels of MMP2 and MMP9, which are closely related to cell invasion. As shown in Fig. 2E and F, miR-142-3p overexpression significantly inhibited the mRNA expressions of MMP2 and MMP9, whereas knockdown of miR-142-3p significantly promoted the expression of MMP2 and MMP9. We further detected the activity of MMP2 and MMP9 by Gelatin zymography. The results showed that miR-142-3p overexpression obviously reduced the gelatinolytic activity of the active form of MMP2 and MMP9, while miR-142-3p inhibition had an opposite result (Fig. $2 \mathrm{G}$ and $\mathrm{H}$ ). These results indicated that miR-142-3p can inhibit invasiveness of trophoblast cells via increased secretion and activity of MMP2 and MMP9.

TGF- $\beta 1$ is a direct target of miR-142-3p. To further elucidate the underlying molecular mechanisms by which miR-142-3p functions in HTR8/SVneo cells, we used the bio-informatic tools (miRBase and TargetScan) to search for the potential targets. To our interest, TGF- $\beta 1$, which has been found to be involved in the invasion of human trophoblast cells (26-29), could bind to miR-142-3p (Fig. 3A). Thus, TGF- $\beta 1$ was selected for further investigation. To experimentally validate whether TGF- $\beta 1$ was a direct target of miR-142-3p, a dual-luciferase reporter assay was conducted. The results showed that overexpression of miR-142-3p significantly decreased the luciferase activity of wt-TGF- $\beta 1-3$ 'UTR, whereas knockdown of miR-142-3p increased luciferase activity. Likewise, cells co-transfected with miR-142-3p mimics, miR-142-3p inhibitor, and TGF- $\beta 1$-mut-3'UTR, showed no obvious change in their luciferase activity (Fig. 3B). In addition, we explored whether miR-142-3p could modulate the expression of TGF- $\beta 1$. As shown in Fig. $3 \mathrm{C}$ and D, the mRNA and protein levels of TGF- $\beta 1$ was decreased after overexpression of miR-142-3p, whereas it increased after inhibition of miR-142-3p. These results suggest that miR-142-3p may affect the invasion and migration of HTR8/ SVneo cells partly at least, by targeting TGF- $\beta 1$.

miR-142-3p inhibition promoted invasiveness of trophoblast cells through the upregulation of TGF- $\beta 1$. Based on the 


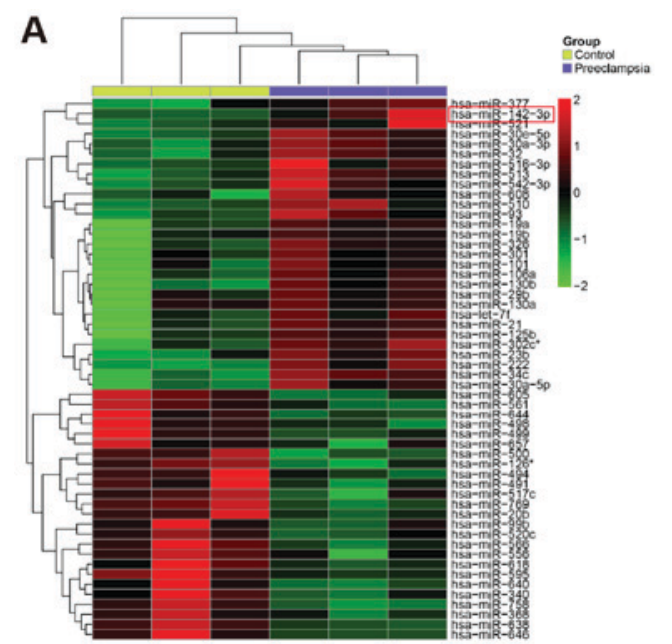

B

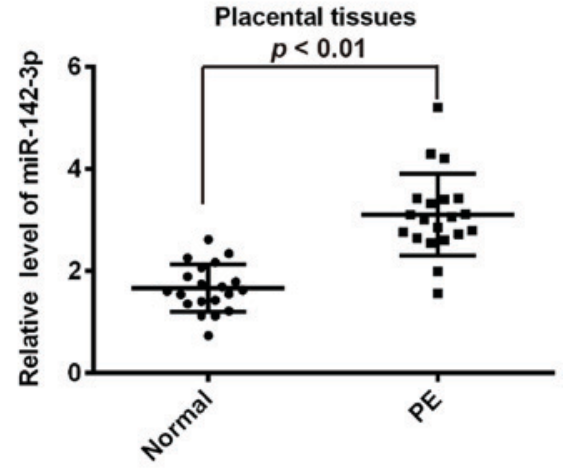

Figure 1. miR-142-3p was upregulated in preeclamptic placentas specimens. (A) Heatmap of the normalized expression levels of miRNAs in placenta samples from 3 patients with PE and 3 women with normal pregnancies. Green indicates low expression levels; red indicates high expression levels. (B) RT-qPCR was performed to determine the expression levels of miR-142-3p in preeclamptic placentas $(n=20)$ and normal pregnancies $(n=20)$. $P<0.01$ vs. Normal group. miRNA/miR, microRNAs; PE, preeclampsia; RT-qPCR, reverse transcription-quantitative polymerase chain reaction.
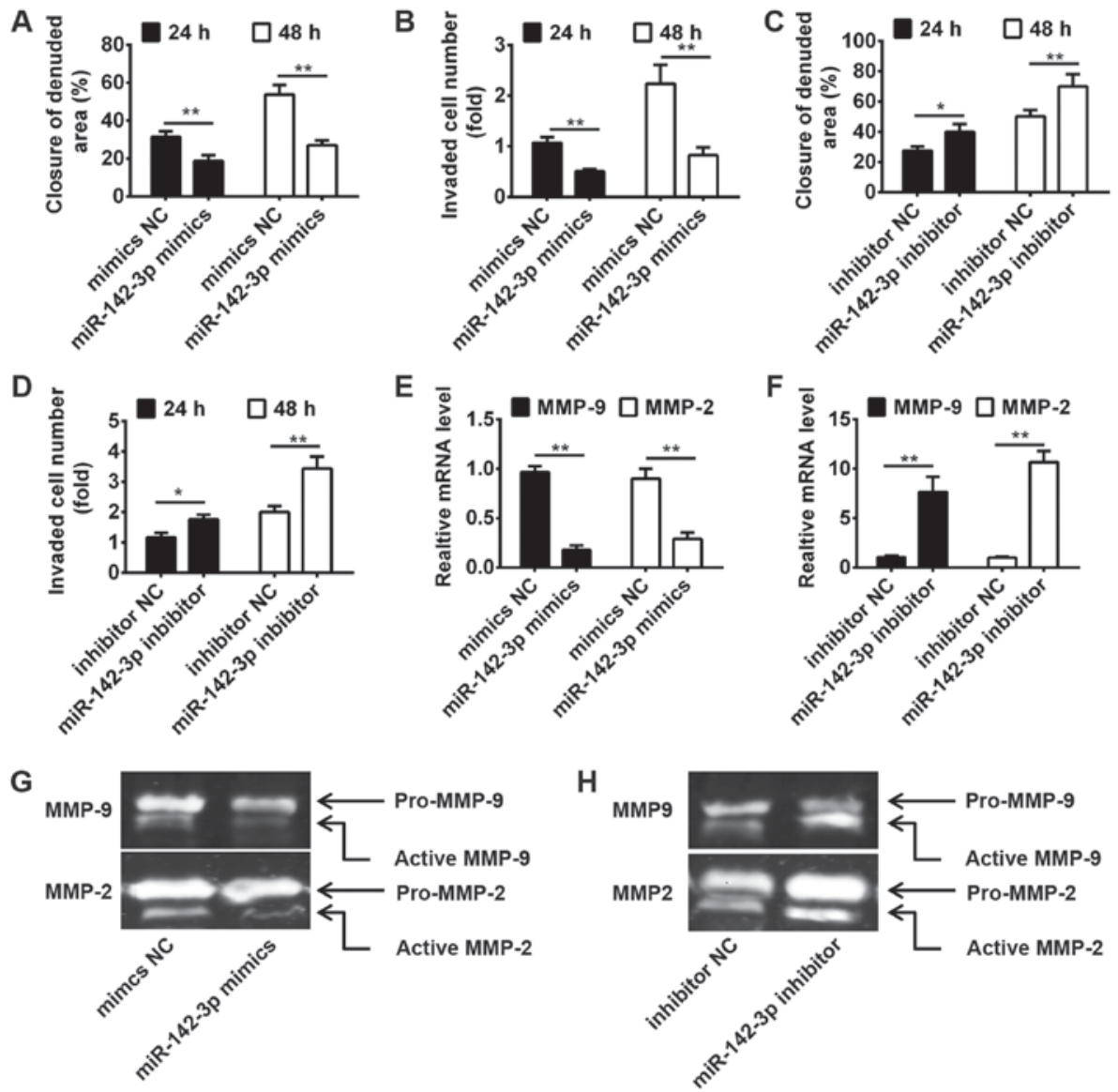

Figure 2. miR-142-3p inhibits the invasion and migration of trophoblast cells. HTR-8/SVneo cells were transfected with miR-142-3p mimics, miR-142-3p inhibitor or miRNA negative controls prior to wound healing and transwell invasion assays. Cell (A) migration and (B) invasion was measured at 24 and $48 \mathrm{~h}$ in cells transfected with mimics. Cell (C) migration and (D) invasion was measured at 24 and $48 \mathrm{~h}$ in cells transfected with inhibitor. The mRNA expression of MMP2 and MMP9 in (E) mimics and (F) inhibitor-transfected cells was analyzed by RT-qPCR. (G and H) The activities of MMP2 and MMP9 were measured by Gelatin zymography. Data are presented as the mean \pm standard deviation of three independent experiments. "P<0.05 and "** $\mathrm{P}<0.01$, as indicated. miRNA/ miR, microRNAs; MMP, matrix metalloproteinase; RT-qPCR, reverse transcription-quantitative polymerase chain reaction; NC, negative control.

findings above, we hypothesized that miR-142-3p inhibitor might promote trophoblast cells invasion and migration by upregulating TGF- $\beta 1$ expression. Firstly, we co-transfected
miR-142-3p inhibitor and TGF- $\beta 1$ siRNA into HTR8/SVneo cells, and the invasion and migration were measured. As shown in Fig. $4 \mathrm{~A}$ and B, si-TGF- $\beta 1$ reversed the promotive effects of 
A
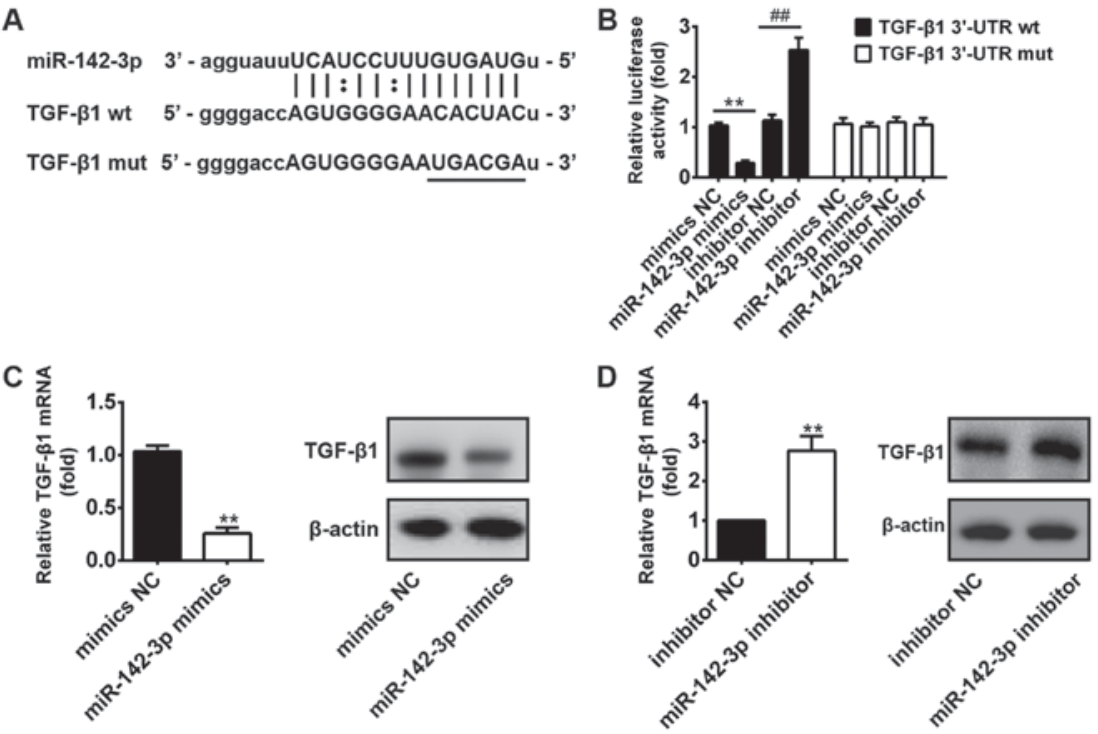

Figure 3. TGF- $\beta 1$ is a direct target of miR-142-3p. (A) The putative binding site of miR-142-3p and TGF- $\beta 1$ is shown. (B) miR-142-3p mimic suppressed the luciferase activities of constructs containing the 3'-UTR segment of TGF- $\beta 1$, while miR-142-3p inhibitor significantly increased the luciferase activities of constructs containing the $3^{\prime}-\mathrm{UTR}$ segment of TGF- $\beta 1(\mathrm{n}=3) .{ }^{* *} \mathrm{P}<0.01$ vs. mimics NC; ${ }^{* \#} \mathrm{P}<0.01$ vs. inhibitor NC. (C and D) The expressions of TGF- $\beta 1 \mathrm{mRNA}$ and protein following transfection with (C) miR-142-3p mimic or (D) miR-142-3p inhibitor were measured by RT-qPCR and western blotting. Data are presented as the mean \pm standard deviation of three independent experiments. ${ }^{* *} \mathrm{P}<0.01$ vs. associated $\mathrm{NC}$ group. miRNA/miR, microRNAs; UTR, untranslated region; TGF, transforming growth factor; wt, wild-type; mut, mutant; RT-qPCR, reverse transcription-quantitative polymerase chain reaction; NC, negative control.

A

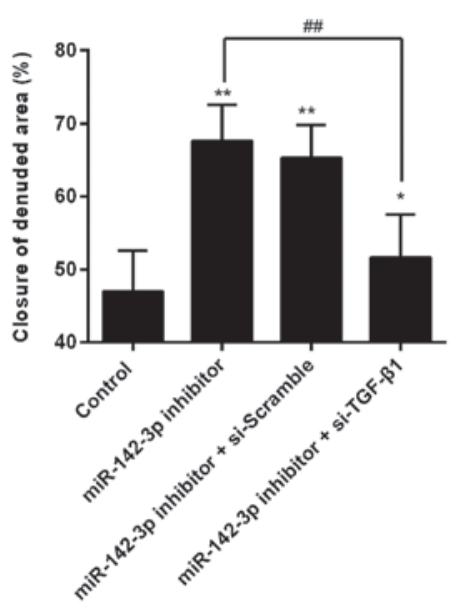

C

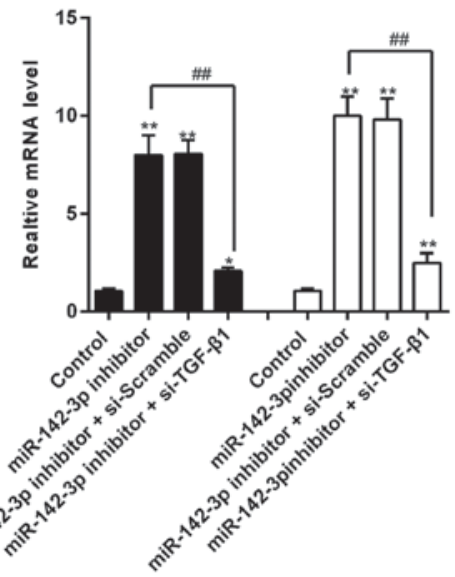

B

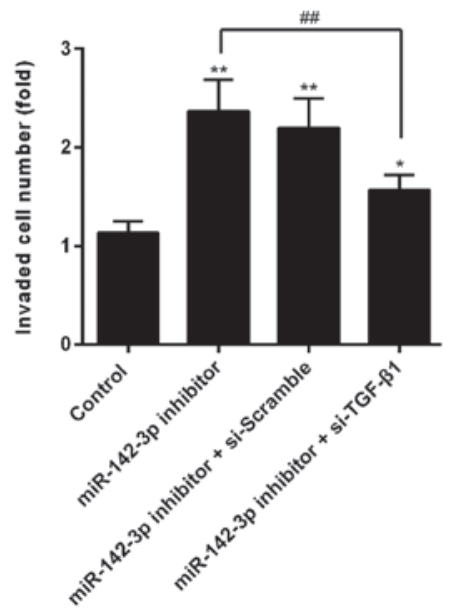

D

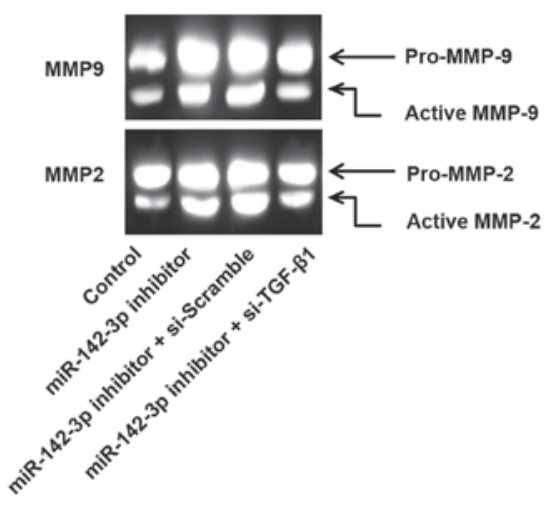

Figure 4. Si-TGF- $\beta 1$ reversed the effect of miR-142-3p inhibitor on cell invasion in HTR8/SVneo cells. HTR8/SVneo cells were co-transfected with si-TGF- $\beta 1$ and miR-142-3p inhibitor. (A) Wound healing assay was performed to monitor cell migration. (B) Transwell assay was performed to monitor cell invasiveness. (C) The mRNA expression of MMP2 and MMP9 was analyzed by RT-qPCR. (D) The activities of MMP2 and MMP9 were measured by Gelatin zymography. Data are presented as the mean \pm standard deviation of three independent experiments. ${ }^{~} \mathrm{P}<0.05$ and ${ }^{* *} \mathrm{P}<0.01$ vs. control group; ${ }^{\# \#} \mathrm{P}<0.01$ vs. miR-142-3p inhibitor. miRNA/miR, microRNAs; UTR, untranslated region; TGF, transforming growth factor; si-, small interfering RNA; MMP, matrix metalloproteinase; RT-qPCR, reverse transcription-quantitative polymerase chain reaction; NC, negative control. 

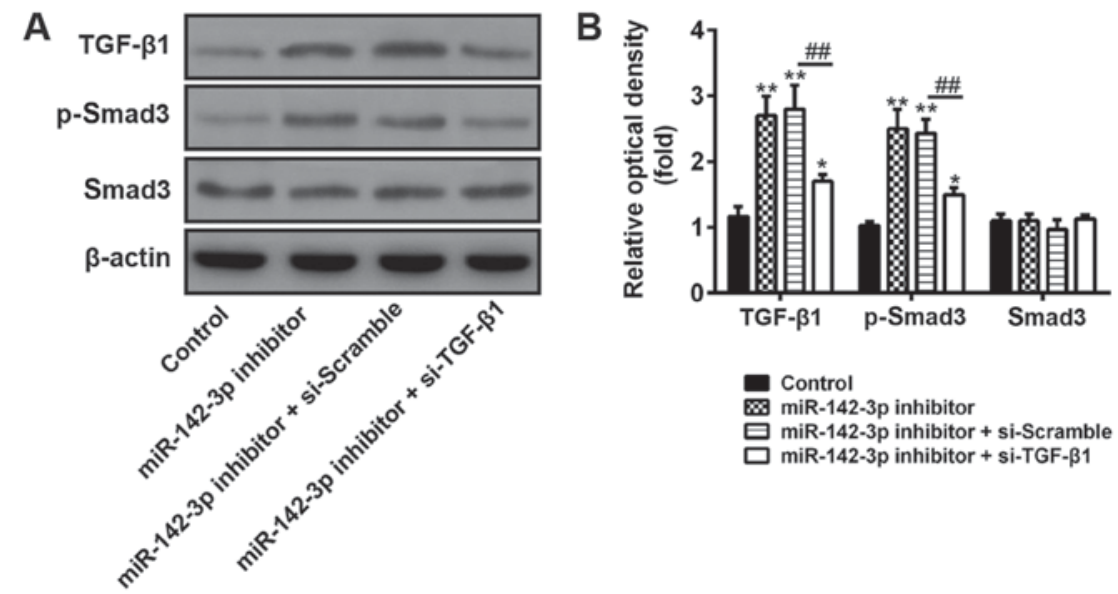

Figure 5. Knockdown of miR-142-3p promoted the activation of the TGF- $\beta 1 / \mathrm{Smad} 3$ signaling pathway. HTR8/SVneo cells were co-transfected with si-TGF- $\beta 1$ and miR-142-3p inhibitor. Following $48 \mathrm{~h}$ transfection, HTR8/SVneo cells were harvested for western blot analysis. (A) The protein expressions of TGF- $\beta 1$, smad 3 and p-smad 3 were detected by western blotting. (B) The bands were semi-quantitatively analyzed using Image J software, normalized to $\beta$-actin density. Data are presented as the mean \pm standard deviation of three independent experiments. ${ }^{~} \mathrm{P}<0.05$ and ${ }^{* *} \mathrm{P}<0.01$ vs. control group; ${ }^{\# \#} \mathrm{P}<0.01$ vs. miR-142-3p inhibitor + si-Scramble group. miRNA/miR, microRNAs; TGF, transforming growth factor; si-, small interfering RNA; p-, phosphorylated.

miR-142-3p on HTR8/SVneo cell invasion and migration. In addition, the increased mRNA expression levels of MMP-2 and MMP-9 were markedly reduced when TGF- $\beta 1$ siRNA transfection (Fig. 4C). Similarly, si-TGF- $\beta 1$ markedly reduced the gelatinolytic activity of the active form of MMP2 and MMP9 mediated by miR-142-3p inhibition (Fig. 4D). These data indicate that miR-142-3p inhibitor promoted invasiveness of trophoblast cells through the upregulation of TGF- $\beta 1$.

Downregulation of miR-142-3p promoted invasiveness of trophoblast cells via activation of TGF- $\beta 1 /$ Smad3 signaling pathway. Previous studies showed that TGF- $\beta 1$ stimulated migration and invasion of various cell types via smad signaling pathway (30-32). Therefore, we hypothesize that a similar pathway might be involved in the action of miR-142-3p on the invasion of trophoblast cells. As indicated in Fig. 5A and B, miR-142-3p inhibitor promoted the phosphorylation level of smad3 (p-smad3) compared with control group. In contrast, when si-TGF- $\beta 1$ was transfected to the miR-142-3p inhibitor group, the expression of p-smad3 became significantly lower. In addition, the total protein of $\operatorname{smad} 3$ has not been affected. This result indicates that miR-142-3p inhibitor promotes the invasion and migration of trophoblast cells via the TGF- $\beta 1 / \operatorname{smad} 3$ signaling pathway.

\section{Discussion}

In the present study, we found that miR-142-3p was upregulated in placentas tissues. Our findings have also demonstrated that miR-142-3p inhibition promoted the invasion and migration of trophoblast cells by regulating the TGF- $\beta 1 / \mathrm{Smad} 3$ signaling pathway. Thus, miR-142-3p/TGF- $\beta 1 / \mathrm{smad} 3$ axis may be a potential target for the prevention and treatment of PE.

Increasing evidence supports that miRNAs play important roles in the development and progression of PE $(7,33,34)$. For example, Tamaru et al (35) found that miR-135b suppressed HTR-8/SVneo cells invasion by directly down regulating chemokine (C-X-C motif) ligand 12 (CXCL12) under low oxygen conditions. Another study from Li et al (36), showed that
miR-125b-1-3p could suppress the invasiveness of trophoblast cells by targeting sphingosine-1-phosphate receptor 1 (S1PR1) in PE. These inspired us to investigate whether there may be other miRNAs involved in the regulation of trophoblastic invasion. In this study, we conducted a microarray miRNA expression analysis to identify miRNA(s) associated with PE, and miR-142-3p was selected as one of miRNAs being most significantly upregulated in placental tissues. Our data imply miR-142-3p may be associated with the pathogenesis of PE.

miR-142-3p was originally identified as a tumor suppressor, which played a crucial role in tumor cell proliferation and invasion $(37,38)$. For example, miR-142-3p was found to be decreased in cervical cancer cells and overexpression of miR-142-3p resulted in downregulation of frizzled7 receptor (FZD7) and inhibited proliferation and invasion in HeLa and SiHa cells (21). Furthermore, Schwickert et al (20) found that miR-142-3p inhibited breast cancer cell invasiveness by synchronous targeting of wiskott-aldrich syndrome-like (WASL) and Integrin Alpha V (ITGAV). However, whether miR-142-3p regulates trophoblast cell function is largely unknown. In the present study, we proved that the overexpression of miR-142-3p inhibited the invasion and migration of HTR-8/SVneo cells, whereas the knockdown of miR-142-3p led to increased invasive and migratory abilities of HTR-8/SVneo cells. According to previous reports, the MMP family plays a crucial role in the digestion of the extracellular matrix, MMP2 and MMP9 are highly expressed in trophoblasts and crucial for trophoblast invasion $(24,25)$. Wang et al (39) showed that Benzo[a] pyrene-7,8-diol-9,10-epoxide (BPDE) suppresses the migration and invasion of human extravillous trophoblast HTR-8/ SVneo cells by downregulating MMP2. Deng et al (40) found that $\mathrm{N}$-acetylglucosaminyltransferase V (MGAT5) inhibited the invasion of trophoblast cells by attenuating MMP2/9 activity in early human pregnancy. However, the significance of MMP2 and MMP9 for miR-142-3p-mediated invasion of trophoblast cells has not been elucidated. Here, we found that the mRNA expressions and the activities of MMP2 and MMP9 was suppressed by overexpression 
of miR-142-3p, but increased by miR-142-3p knockdown. These finding indicate that miR-142-3p could suppress the invasiveness of trophoblast cells via increased secretion and activity of MMP2 and MMP9. However, the molecular mechanisms behind the regulation of miR-142-3p expression and its role in the trophoblast invasion remain unknown.

Transforming growth factor (TGF)- $\beta 1$ is a member of the TGF superfamily, which has been shown to be a multifunctional cytokine required for embryonic development and regulation of trophoblast cell behaviors (41). Increasing evidence demonstrated that TGF- $\beta 1$ could inhibit trophoblast cell proliferation and invasion/migration in some of the transformed trophoblast cell lines, including HRT-8/ SVneo, SGHP-4, and ED-27 (42-44). A recent research from Karmakar et al (45), showed that TGF- $\beta 1$ mediated upregulation of cell-to-cell adhesion with reduced cell-to-matrix interaction along with an increased ezrin and E-cadherin expression, is associated with reduced invasiveness of trophoblast cells. Interestingly, previous study reported that miRNAs play important roles in the regulation of cancer metastasis via regulating TGF- $\beta 1$ directly or indirectly $(46,47)$. Thus, we speculated that whether the TGF- $\beta 1$ is involved in miR-142-3p mediated inhibiting effect on the invasion of trophoblast cells. In the present study, bioinformatics was used to predict the target genes that regulate by miR-142-3p and it was determined that TGF- $\beta 1$ may be closely associated with miR-142-3p. Our study also revealed that the TGF- $\beta / \mathrm{Smad} 3$ signaling pathway was modulated by miR-142-3p. TGF- $\beta / \mathrm{Smad} 3$ signaling pathway play a crucial role in the invasion of human JEG-3 trophoblast cells (48). It is well known that the downstream effects of TGF- $\beta 1$ are mediated by smads, a family of intracellular transcription factors (49). We assumed that knockdown of miR-142-3p can increase expression of TGF- $\beta 1$, thus promoting smad 3 changing to $\mathrm{p}$-smad 3 in trophoblast cells. In contrast, si-TGF- $\beta 1$ reversed the promoting effect of miR-142-3p knockdown on the activity of TGF- $\beta / \mathrm{Smad} 3$ signaling. Based on these findings, we suggest that inhibition of $\mathrm{miR}-142-3 \mathrm{p}$ promotes the activation of $\mathrm{TGF}-\beta 1 / \mathrm{Smad} 3$ signaling, and subsequently enhances the invasion of trophoblast cells.

In conclusion, we demonstrated that miR-142-3p is highly expressed in preeclamptic placentas specimens. Furthermore, our study provides preliminary evidence that inhibition of miR-142-3p promotes the invasion of trophoblast cells via TGF- $\beta 1 /$ smad3 pathway. Therefore, miR-142-3p/TGF- $\beta 1 /$ Smad3 axis may be developed to be a potential therapeutic target for PE.

\section{Acknowledgements}

Not applicable.

\section{Funding}

No funding was received.

\section{Availability of data and materials}

All data generated or analyzed during the present study are included in this published article.

\section{Authors' contributions}

EL performed the experiments, contributed to data analysis and wrote the paper. EL, ZL, YZ, MC, LW and JL analyzed the data. ZL conceptualized the study design, and contributed to the data analysis and experimental materials. All authors read and approved the final manuscript.

\section{Ethics approval and consent to participate}

All experimental protocols were approved by the Ethics Committee of the Tangshan Worker Hospital, He Bei Medical University (Hebei, China). Written informed consent was obtained from all patients.

\section{Patient consent for publication}

Not applicable.

\section{Competing interests}

The authors declare that they have no competing interests.

\section{References}

1. Redman CW: Current topic: Pre-eclampsia and the placenta. Placenta 12: 301-308, 1991.

2. Kanasaki K and Kalluri R: The biology of preeclampsia. Kidney Int 76: 831-837, 2009.

3. Moffett A and Loke C: Immunology of placentation in eutherian mammals. Nat Rev Immunol 6: 584-594, 2006.

4. Noris M, Perico N and Remuzzi G: Mechanisms of disease: Pre-eclampsia. Nat Clin Pract Nephrol 1: 98-114; quiz 120, 2005.

5. Fisher SJ: The placental problem: Linking abnormal cytotrophoblast differentiation to the maternal symptoms of preeclampsia. Reprod Biol Endocrinol 2: 53, 2004.

6. Wang A, Rana S and Karumanchi SA: Preeclampsia: The role of angiogenic factors in its pathogenesis. Physiology (Bethesda) 24: $147-158,2009$

7. Yu Y, Wang L, Liu T and Guan H: MicroRNA-204 suppresses trophoblast-like cell invasion by targeting matrix metalloproteinase-9. Biochem Biophys Res Commun 463: 285-291, 2015.

8. Moindjie H, Santos ED, Loeuillet L, Gronier H, de Mazancourt P, Barnea ER, Vialard F and Dieudonne MN: Preimplantation factor (PIF) promotes human trophoblast invasion. Biol Reprod 91: 118, 2014.

9. Bartel DP: MicroRNAs: Genomics, biogenesis, mechanism, and function. Cell 116: 281-297, 2004.

10. Kloosterman WP and Plasterk RH: The diverse functions of microRNAs in animal development and disease. Dev Cell 11: 441-450, 2006.

11. Tu K, Liu Z, Yao B, Han S and Yang W: MicroRNA-519a promotes tumor growth by targeting PTEN/PI3K/AKT signaling in hepatocellular carcinoma. Int J Oncol 48: 965-974, 2016.

12. Hu Y, Li P, Hao S, Liu L, Zhao J and Hou Y: Differential expression of microRNAs in the placentae of Chinese patients with severe pre-eclampsia. Clin Chem Lab Med 47: 923-929, 2009.

13. Zhu XM, Han T, Sargent IL, Yin GW and Yao YQ: Differential expression profile of microRNAs in human placentas from preeclamptic pregnancies vs normal pregnancies. Am J Obstet Gynecol 200: 661.e1-7, 2009.

14. Enquobahrie DA, Abetew DF, Sorensen TK, Willoughby D, Chidambaram K and Williams MA: Placental microRNA expression in pregnancies complicated by preeclampsia. Am J Obstet Gynecol 204: 178.e12-21, 2011.

15. Livak KJ and Schmittgen TD: Analysis of relative gene expression data using real-time quantitative PCR and the 2(-Delta Delta C(T)) method. Methods 25: 402-408, 2001.

16. Nadeem L, Munir S, Fu G, Dunk C, Baczyk D, Caniggia I, Lye S and Peng C: Nodal signals through activin receptor-like kinase 7 to inhibit trophoblast migration and invasion: Implication in the pathogenesis of preeclampsia. Am J Pathol 178: 1177-1189, 2011. 
17. Yang S, Li H, Ge Q, Guo L and Chen F: Deregulated microRNA species in the plasma and placenta of patients with preeclampsia. Mol Med Rep 12: 527-534, 2015.

18. Betoni JS, Derr K, Pahl MC, Rogers L, Muller CL, Packard RE, Carey DJ, Kuivaniemi H and Tromp G: MicroRNA analysis in placentas from patients with preeclampsia: Comparison of new and published results. Hypertens Pregnancy 32: 321-339, 2013.

19. Song J, Li Y and An RF: Identification of early-onset preeclampsia-related genes and MicroRNAs by bioinformatics approaches. Reprod Sci 22: 954-963, 2015.

20. Schwickert A, Weghake E, Brüggemann K, Engbers A, Brinkmann BF, Kemper B, Seggewiß J, Stock C, Ebnet K, Kiesel L, et al: microRNA miR-142-3p inhibits breast cancer cell invasiveness by synchronous targeting of WASL, Integrin Alpha $\mathrm{V}$ and additional cytoskeletal elements. PLoS One 10: e0143993, 2015.

21. Deng B, Zhang Y, Zhang S, Wen F, Miao Y and Guo K: MicroRNA-142-3p inhibits cell proliferation and invasion of cervical cancer cells by targeting FZD7. Tumour Biol 36: 8065-8073, 2015.

22. Xu G, Wang J, Jia Y, Shen F, Han W and Kang Y: MiR-142-3p functions as a potential tumor suppressor in human osteosarcoma by targeting HMGA1. Cell Physiol Biochem 33: 1329-1339, 2014.

23. Mandl M, Haas J, Bischof P, Nöhammer G and Desoye G: Serum-dependent effects of IGF-I and insulin on proliferation and invasion of human first trimester trophoblast cell models. Histochem Cell Biol 117: 391-399, 2002.

24. Staun-Ram E, Goldman S, Gabarin D and Shalev E: Expression and importance of matrix metalloproteinase 2 and 9 (MMP-2 and -9) in human trophoblast invasion. Reprod Biol Endocrinol 2: 59,2004

25. Xu P, Alfaidy $\mathrm{N}$ and Challis JR: Expression of matrix metalloproteinase (MMP)-2 and MMP-9 in human placenta and fetal membranes in relation to preterm and term labor. J Clin Endocrinol Metab 87: 1353-1361, 2002.

26. Li Y, Zhu H, Klausen C, Peng B and Leung PC: Vascular endothelial growth factor-A (VEGF-A) mediates activin A-induced human trophoblast endothelial-like tube formation. Endocrinology 156: 4257-4268, 2015.

27. Belkacemi L, Lash GE, Macdonald-Goodfellow SK, Caldwell JD and Graham $\mathrm{CH}$ : Inhibition of human trophoblast invasiveness by high glucose concentrations. J Clin Endocrinol Metab 90 : 4846-4851, 2005.

28. Fitzgerald JS, Germeyer A, Huppertz B, Jeschke U, Knöfler M, Moser G, Scholz C, Sonderegger S, Toth B and Markert UR: Governing the invasive trophoblast: Current aspects on intraand extracellular regulation. Am J Reprod Immunol 63: 492-505, 2010.

29. Knöfler M: Critical growth factors and signalling pathways controlling human trophoblast invasion. Int J Dev Biol 54 269-280, 2010.

30. Xiong S, Cheng JC, Klausen C, Zhao J and Leung PC: TGF- $\beta 1$ stimulates migration of type II endometrial cancer cells by down-regulating PTEN via activation of SMAD and ERK1/2 signaling pathways. Oncotarget 7: 61262-61272, 2016

31. Attisano L and Wrana JL: Signal transduction by the TGF-beta superfamily. Science 296: 1646-1647, 2002.

32. Ungefroren H, Sebens S, Giehl K, Helm O, Groth S, Fändrich F, Röcken C, Sipos B, Lehnert H and Gieseler F: Raclb negatively regulates TGF- $\beta 1$-induced cell motility in pancreatic ductal epithelial cells by suppressing Smad signalling. Oncotarget 5: 277-290, 2014
33. Luo L, Ye G, Nadeem L, Fu G, Yang BB, Honarparvar E, Dunk C, Lye S and Peng C: MicroRNA-378a-5p promotes trophoblast cell survival, migration and invasion by targeting Nodal. J Cell Sci 125: 3124-3132, 2012

34. Bai Y, Yang W, Yang HX, Liao Q, Ye G, Fu G, Ji L, Xu P, Wang H, Li YX, et al: Downregulated miR-195 detected in preeclamptic placenta affects trophoblast cell invasion via modulating ActRIIA expression. PLoS One 7: e38875, 2012.

35. Tamaru S, Mizuno Y, Tochigi H, Kajihara T, Okazaki Y, Okagaki R, Kamei Y, Ishihara O and Itakura A: MicroRNA-135b suppresses extravillous trophoblast-derived HTR-8/SVneo cell invasion by directly down regulating CXCL12 under low oxygen conditions. Biochem Biophys Res Commun 461: 421-426, 2015.

36. Li Q, Pan Z, Wang X, Gao Z, Ren C and Yang W: miR-125b-1-3p inhibits trophoblast cell invasion by targeting sphingosine-1-phosphate receptor 1 in preeclampsia. Biochem Biophys Res Commun 453: 57-63, 2014.

37. Zhang J, Shan WF, Jin TT, Wu GQ, Xiong XX, Jin HY and Zhu SM: Propofol exerts anti-hepatocellular carcinoma by microvesicle-mediated transfer of miR-142-3p from macrophage to cancer cells. J Transl Med 12: 279, 2014.

38. Chai S, Tong M, Ng KY, Kwan PS, Chan YP, Fung TM, Lee TK, Wong N, Xie D, Yuan YF, et al: Regulatory role of miR-142-3p on the functional hepatic cancer stem cell marker CD133. Oncotarget 5: 5725-5735, 2014.

39. Wang R, Wang W, Ao L, Wang Z, Hao X and Zhang H: Benzo[a] pyrene-7,8-diol-9,10-epoxide suppresses the migration and invasion of human extravillous trophoblast HTR-8/SVneo cells by down-regulating MMP2 through inhibition of FAK/SRC/PI3K/ AKT pathway. Toxicology 386: 72-83, 2017.

40. Deng Q, Chen Y, Yin N, Shan N, Luo X, Tong C, Zhang H, Baker PN, Liu X and Qi H: N-acetylglucosaminyltransferase V inhibits the invasion of trophoblast cells by attenuating MMP2/9 activity in early human pregnancy. Placenta 36: 1291-1299, 2015.

41. Ayatollahi M, Geramizadeh B, Yazdani M and Azarpira N: Effect of the immunoregulatory cytokines on successful pregnancy depends upon the control of graft rejection mechanisms. Transplant Proc 39: 244-245, 2007.

42. Luo S, Yu H, Wu D and Peng C: Transforming growth factor-betal inhibits steroidogenesis in human trophoblast cells. Mol Hum Reprod 8: 318-325, 2002.

43. Ma Y, Ryu JS, Dulay A, Segal M and Guller S: Regulation of plasminogen activator inhibitor (PAI)-1 expression in a human trophoblast cell line by glucocorticoid (GC) and transforming growth factor (TGF)-beta. Placenta 23: 727-734, 2002

44. Tse WK, Whitley GS and Cartwright JE: Transforming growth factor-betal regulates hepatocyte growth factor-induced trophoblast motility and invasion. Placenta 23: 699-705, 2002.

45. Karmakar S and Das C: Regulation of trophoblast invasion by IL-1beta and TGF-betal. Am J Reprod Immunol 48: 210-219, 2002

46. Li Q, Cheng Q, Chen Z, Peng R, Chen R, Ma Z, Wan X, Liu J, Meng M, Peng Z and Jiang B: MicroRNA-663 inhibits the proliferation, migration and invasion of glioblastoma cells via targeting TGF- $\beta 1$. Oncol Rep 35: 1125-1134, 2016.

47. Liu ZY, Zhang GL, Wang MM, Xiong YN and Cui HQ MicroRNA-663 targets TGFB1 and regulates lung cancer proliferation. Asian Pac J Cancer Prev 12: 2819-2823, 2011.

48. Huang Z, Li S, Fan W and Ma Q: Transforming growth factor $\beta 1$ promotes invasion of human JEG-3 trophoblast cells via TGF- $\beta$ / Smad3 signaling pathway. Oncotarget 8: 33560-33570, 2017.

49. Heldin $\mathrm{CH}$, Miyazono K and ten Dijke P: TGF-beta signalling from cell membrane to nucleus through SMAD proteins. Nature 390: 465-471, 1997. 